\title{
Tilted vortices in a superconducting mesoscopic cylinder
}

\author{
Antonio R. de C. Romaguera, ${ }^{1,2, *}$ Mauro M. Doria, ${ }^{1,2, \dagger}$ and F. M. Peeters ${ }^{1, \$}$ \\ ${ }^{1}$ Departement Fysica, Universiteit Antwerpen, Groenenborgerlaan 171, B-2020 Antwerpen, Belgium \\ ${ }^{2}$ Universidade Federal do Rio de Janeiro, C. P. 68.528, 21941-972 Rio de Janeiro, Brazil
}

(Received 29 December 2006; published 24 May 2007)

\begin{abstract}
Vortex patterns are determined for a superconducting cylinder with mesoscopic dimensions, namely, larger than the coherence length and smaller than the London penetration length, using the nonlinear GinzburgLandau theory. The critical fields and matching fields are obtained for field orientation parallel and perpendicular to the cylinder axis. In the latter case, vortex rearrangements are found to occur in the vortex patterns. Tilting of the field leads to interesting phenomena caused by the small volume-to-surface ratio, as vortex lines are preferentially oriented along the field direction while they are forced to be perpendicular to the surface. Vortices can enter and leave the cylinder also by simply rotating the applied field and we study here in detail the cases up to three vortices. We find the striking result of a single vortex with the lowest free-energy configuration achieved at a tilted angle.
\end{abstract}

DOI: $10.1103 /$ PhysRevB.75.184525

PACS number(s): 74.78.Na, 74.25.-q, 74.20.De

\section{INTRODUCTION}

Mesoscopic superconductors exhibit very peculiar magnetic properties when their size is comparable to the coherence length $\xi$. The magnetic properties of very thin superconducting disks have been experimentally ${ }^{1,2}$ measured for several radii and their magnetic properties are in agreement with theoretical studies. ${ }^{3,4}$ For a radius $R$ of the order of, or less than, $\xi$, no vortices can enter and only the Meissner state is possible. Above this limit, and for $R<2 \xi$, giant vortex states are allowed, while for $R>2 \xi$ multivortex states become possible, as reported in Ref. 5 and recently observed in Refs. 6 and 7. Theoretical studies have been done in the context of the phenomenological Ginzburg-Landau theory, able to predict many novel properties of the vortex patterns in mesoscopic superconductors not found in the bulk. For example, in a thin superconducting square with side $2 \lambda$, it was theoretically predicted ${ }^{8}$ that the vortex pattern symmetry follows the $O_{4}$ group, resulting in induced antivortices for certain values of the applied field. ${ }^{9}$ However, these studies are limited to the so-called thin thickness limit with the applied field oriented perpendicularly to the flat geometry. In such situations the sample thickness $D$ is much smaller than the coherence length, $D \ll \xi$, the complex order parameter is integrated along the thinnest direction reducing the problem to a flat surface, and consequently, to a lower dimensionality.

The thin disk under the condition $D \ll \lambda$ was intensively studied. ${ }^{3,4}$ Beyond the thin disk limit, the wirelike geometry $^{10}$ and samples with a constriction ${ }^{11}$ were considered with field oriented parallel to the cylinder major axis, a problem that in some limits is discussed in text books. ${ }^{12,13}$ The thin-film limit was also applied to the study of hollow spheres. ${ }^{14}$ The fact that all these studies rely on some dimensional reduction of the problem prevents them from addressing some interesting questions associated to the truly threedimensional properties of the superconductor. For instance, some mixed-state properties vary according to field orientation with respect to sample geometry, and vortex patterns stable under some field orientation may be unstable under another orientation.
In this paper we investigate three-dimensional mesoscopic superconductors. We consider two different mesoscopic cylinders with distinct diameter-to-thickness ratios situated between the limits of a disk and a long cylinder. Both have the same radius $R=4.0 \xi$ but different heights, namely, $D=4.0 \xi$ and $8.0 \xi$. Hereafter, we refer to them as short-ratio and equal-ratio cylinders, because they respectively have height to diameter aspect ratio less than and equal to 1 . Their thickness falls in the limit $D>\xi$, and for an applied field not oriented along the cylinder's major axis, it is not possible to use symmetry arguments to determine the vortex patterns. Thus a cylinder under an arbitrarily tilted field demands a genuine three-dimensional treatment of the Ginzburg-Landau equation, and of its three-dimensional borders through the insulator-superconductor boundary condition. Figure 1 shows a pictorial view of a cylinder under an arbitrarily tilted field, where the rotation angle $\theta$ is measured with respect to the major $(z)$ axis: $H=H_{0} \cos \theta$. For $\theta=0^{\circ}$ the comparison with the well-known studied case of a very thin disk is possible. ${ }^{3,15}$ The plane of rotation is referred to as the $y-z$ plane, and we introduce the rotation for fields oriented parallel and perpendicular to the major axis: $H_{\|} \equiv H\left(\theta=0^{\circ}\right)$ and $H_{\perp} \equiv H\left(\theta=90^{\circ}\right)$, respectively.

We will determine several properties of vortex patterns for equal-ratio and short-ratio cylinders, in the case of field orientations $H_{\|}$and $H_{\perp}$. We report on matching fields, magnetization, and free-energy curves for $H_{\perp}$. We find structural vortex transitions in the case of an equal-ratio cylinder that consist of a sudden rearrangement of vortex positions inside the cylinder. In the case of a tilted field we restrict the present discussion to the case of zero, one, two, and three vortices inside the short-ratio cylinder. These field regimes
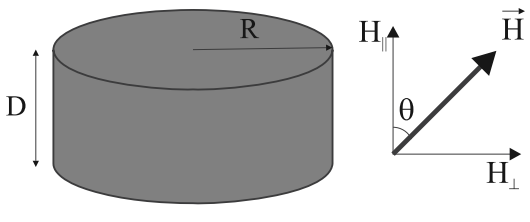

FIG. 1. Schematic view of the system. 
are rich in interesting phenomena, due to the three dimensionality of the system. Their field windows, $\Delta H_{\|}$and $\Delta H_{\perp}$, are quite distinct and this leads to different transitions upon field rotation. Even the Meissner phase has distinct properties in both field orientations, as shown by a fitting function obtained here. A well-known fact is that vortices emerge perpendicularly to the surface. ${ }^{16}$ Simultaneously they tend to remain oriented along the applied field. For a mesoscopic system these two demands are conflicting as surface and volume effects become comparable. We have obtained the length of a vortex line directly from our numerical simulations and compared it to simple models, and found that surface effects are fundamental in determining the total vortex length. Curiously, we found in the short-ratio cylinder that for some fixed applied field the tilted $\left(\theta=43^{\circ}\right)$ single vortex configuration has lower energy than the straight vortex oriented along the major axis $\left(\theta=0^{\circ}\right)$ which also corresponds to a local-energy minimum.

This paper is organized as follows. In Sec. II we present the theoretical approach and discuss the numerical procedure. In Sec. III we study and characterize the vortex patterns for short-ratio and equal-ratio cylinders in the case of two special orthogonal applied field directions, namely the parallel $\left(\theta=0^{\circ}\right)$ and the perpendicular $\left(\theta=90^{\circ}\right)$ orientations. We obtain the critical fields, the matching fields, and the field range that renders a particular vortex pattern as the actual ground state. In the next section, Sec. IV, we analyze the general case of an oblique field for the short-ratio cylinder in the special case of zero-to-zero (Sec. IV A), one-to-zero (Sec. IV B), one-to-one (Sec. IV C), two-to-one (Sec. IV D), three-to-one (Sec. IV E), and three-to-two (Sec. IV F) vortex transitions. In the following section, Sec. V, we analyze the length of one tilted vortex and compare its growth with simplified models. In Sec. VI we conclude and summarize our results.

\section{THEORETICAL APPROACH}

Our theoretical study of the superconducting mesoscopic cylinder, immersed in an insulating medium, and under an oblique field is done using the full three-dimensional Ginzburg-Landau approach (see Refs. 17 and 18 for more details). We will assume that the penetration depth is longer than the sample dimensions, i.e., $\lambda>D, R$, which is satisfied for strong type-II superconductors. Within this approximation we can neglect the screening currents in the system. Under this approximation the Ginzburg-Landau equation stems from minimization of the following free-energy functional with respect to the order parameter $\psi$ :

$$
\mathcal{F}_{c}=\int \frac{d v}{V}\left[-\tau|\psi|^{2}+\frac{1}{2}|\psi|^{4}+\tau \xi^{2}\left|\left(\vec{\nabla}-\frac{2 \pi i}{\Phi_{0}} \vec{A}\right) \psi\right|^{2}\right] .
$$

The three-dimensional continuous $\tau(\vec{r})$ function is equal to 1 inside the superconductor and 0 outside. This functional includes the boundary condition because the $\tau(\vec{r})$ function, multiplied by the covariant derivative, yields the so-called de Gennes boundary condition for the superconductor, $\hat{n} \cdot[\vec{\nabla}$ $\left.-\left(2 \pi i \vec{A} / \Phi_{0}\right)\right]\left.\psi\right|_{s}=0$, valid on the three-dimensional surface. The cylinder is surrounded by an insulating medium and both media are inside a unit-cell box where minimization of the free energy is done with respect to $\psi$. We $\operatorname{chose}^{17}$ a representation for $\tau(\vec{x})$ that connects smoothly the cylinder to the insulator over a distance that is a fraction of $\xi$. Other choices of the $\tau$ function are possible and all render very similar results as shown in Ref. 19. This free energy is expressed in units of the condensate energy, $H_{c}^{2} / 8 \pi$; lengths are in units of the coherence length $\xi$ and $\psi$ scaled by $\sqrt{-\alpha / \beta}$. We choose to express all our field results in units of $H_{\text {ref }}$ $\equiv H_{c 3}^{\| s}$, the maximum possible field for the short cylinder, in the case of parallel orientation. The numerical simulations are done in a discrete version of the theory, using finite differences for the derivatives, such that gauge invariance is kept in the discrete theory. A cubic mesh, typically of size $48 \times 48 \times 48$, is used and the free energy minimized through the simulated annealing technique. We start at an initial field value, taking a random initial configuration for $\psi$, and do our field sweep sequentially, meaning that a previous field value solution is taken as the initial configuration for the next field.

\section{PARALLEL AND PERPENDICULAR FIELDS}

In the present section we discuss the effect of a field applied either parallel or perpendicular to the cylinder axis. We consider the short-ratio and equal-ratio cylinders and find that their vortex patterns differ considerably for $H_{\perp}$ but not for $H_{\|}$. The different heights of the two cylinders ${ }^{1}$ do not produce significant effects under $H_{\|}$, as expected, but do have a pronounced effect under $H_{\perp}$.

For both cases we perform a full field loop sweep, starting from zero and increasing the field to its maximum, $H_{c 3}$, where the normal phase is reached. The loop is closed by decreasing it from $H_{c 3}$ back to zero field again. This procedure allows us to observe the hysteretic behavior. A general noticeable fact ${ }^{20}$ is that for increasing field the numerical procedure always finds the lowest energy whereas for decreasing field often it finds a high-energy state. This is a consequence of the Bean-Livingston surface barrier that exists even in the case of an extreme type-II superconductor. The Bean-Livingston surface barrier stems from two competing effects, usually taken as the magnetic-field shielding (Meissner effect), that pushes the vortex towards the center of the sample and the image vortex, located outside the sample, with opposite vorticity that attracts the vortex to the edge. Here the magnetic-field shielding is not taken into account but there are screening currents, leading to the BeanLivingston barrier. The screening currents at the edge of the sample and the currents around the vortex flow in opposite directions and this makes vortices seek the center of the sample for increasing field, but not for decreasing field. In the latter case the Bean-Livingston barrier is weakened by the surplus of vortices already inside the sample, and so, unable to act effectively. Consequently high-energy states are accessible since vortices are arranged away from the fundamental state. This hysteretic behavior was discussed in several previous papers ${ }^{20,21}$ in the case of a flat surface $\left(H_{\|}\right)$and here is also found to exist for the lateral surface of a cylinder 


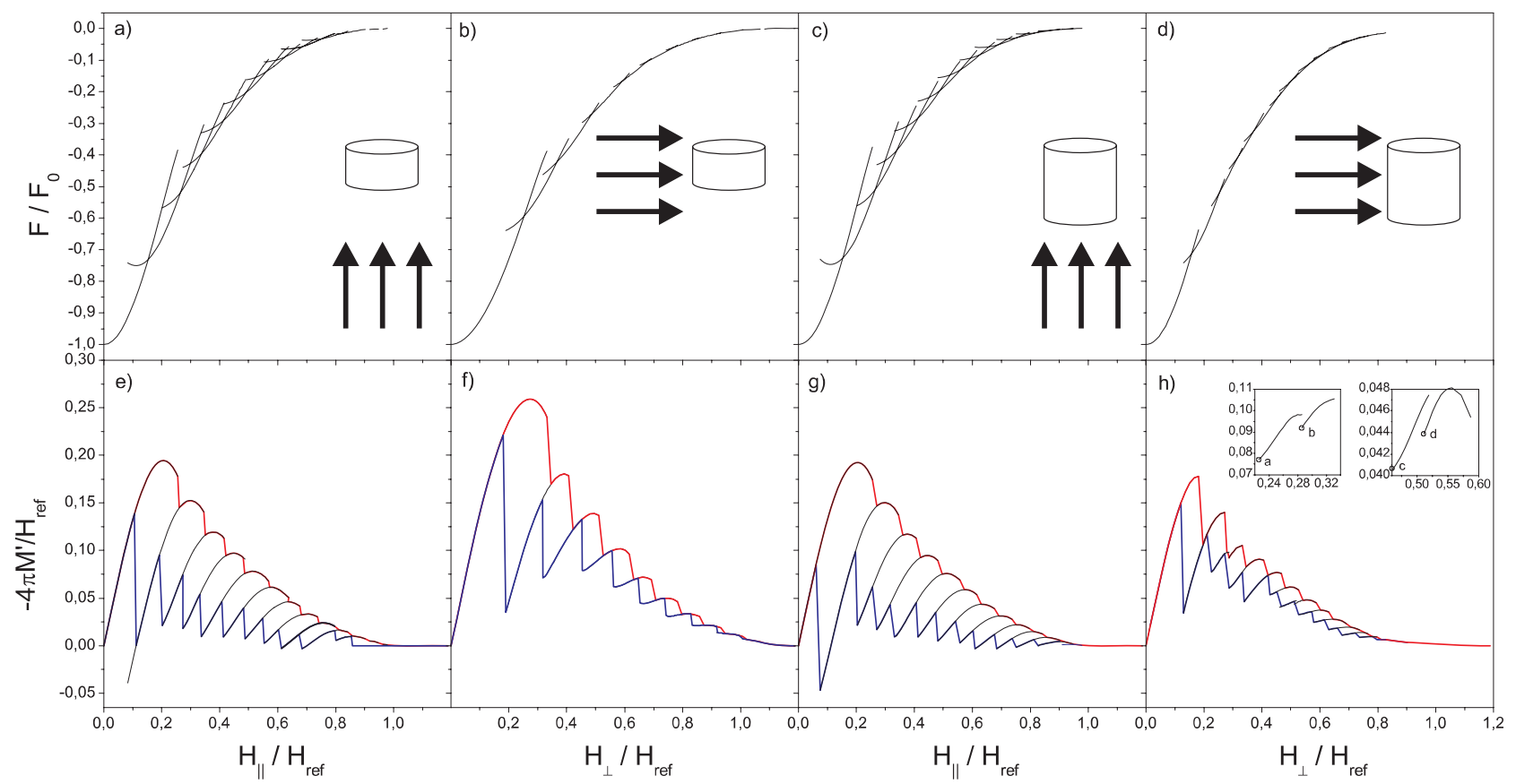

FIG. 2. (Color online) Top figures (a)-(d) show the free energy vs applied field for the short-ratio $(D=4.0 \xi)$ and the equal-ratio $(D$ $=8.0 \xi$ ) cylinders with radius $R=4.0 \xi$. A pictorial view of the cylinder and field orientation are shown as insets. Bottom figures (f) $-(\mathrm{h})$ display the corresponding magnetization curves. $M^{\prime}$ refers to either $D_{y y} M_{y}$ and to $D_{z z} M_{z}$ in the case of $H_{\perp}$ or $H_{\|}$, respectively. The insets in Fig. 2(h) are enlargements of the magnetization curves that show the discontinuity associated to the structural transition observed for $L=2$ (left) and $L=6$ (right).

$\left(H_{\perp}\right)$. Throughout this paper we refer to the Meissner phase as the regime without vortices.

The free-energy vs applied field curves for both cases, $H_{\perp}$ and $H_{\|}$, show the same qualitative structure, a fact that demands some consideration. Both curves are a sum of independent intersecting lines, labeled according to the topological number, or vorticity, $L$, defined as the number of times the phase of the order parameter winds around $2 \pi$ as ones encircles the maximum area. ${ }^{22}$ For $H_{\|}$the topological number is just the total angular momentum, but for $H_{\perp}$ the situation is more complicated as there are vortices of different lengths involved in the problem. Vortices in the center are longer than those at the edges. This offers no difficulty to our numerical approach as we obtain the vortex patterns without invoking any particular order-parameter decomposition. Alternative methods, useful to describe the field evolution between states through the saddle-point approach, ${ }^{5,23,24}$ are not applicable here, because they express the order parameter as a linear combination of angular momentum eigenstates. The angular momentum is not a good quantum number for $H_{\perp}$ as there is no rotational symmetry in this case. However, we chose to use the same notation $L$ to label states for both $H_{\perp}$ and $H_{\|}$cases. The transitions between states with vorticities $L$ and $L+1$ are first order, and these states cross each other at the matching fields $H_{L}$. Formally, in these points $F\left(H_{L}\right)$ $=F\left(H_{L+1}\right)$ and they correspond to a first-order transition.

First, the geometrical properties of the cylinder will be discussed in order to understand its consequences on the vortex patterns for $H_{\perp}$ and $H_{\|}$. Under parallel orientation any rotation around the $z$ axis is an operation of symmetry but for perpendicular orientation the only invariance left is a $\pi$ ro- tation around the $y$ axis. Consequently the density $|\psi|^{2}$ for $H_{\perp}$ is quite different near the two tangent surfaces, namely, the bottom-top and the lateral surfaces. Notice that $H_{\perp}$ is tangential to the whole bottom-top areas but only to two straight lines of the lateral surface. We find that the density $|\psi|^{2}$ shows a strong depletion near the lateral surface.

The free-energy vs applied field curves are plotted in Fig. 2 for the short-ratio and for the equal-ratio cylinders. Figures 2(a) and 2(c) [Figs. 2(b) and 2(d)] show the free energy for $H_{\|}\left[H_{\perp}\right]$ for the equal-ratio and short-ratio cylinders, respectively. For comparison we use as our field unit the maximum field along the parallel direction for the short-ratio cylinder, previously introduced, to express the other critical fields. We found that $H_{c 3}^{\perp s} / H_{r e f}=1.17, H_{c 3}^{\| l} / H_{r e f}=1.00$, and $H_{c 3}^{\perp l} / H_{r e f}$ =1.16. As expected, $H_{c 3}^{\|}$is independent of the vortex length, and so of the height of the cylinder. For the short-ratio cylinder the maximum vorticity $L_{\max }\left(L=0, \ldots, L_{\max }\right)$ is found equal to 12 (parallel) and 10 (perpendicular), whereas for the equal-ratio cylinder it is 12 for both parallel and perpendicular orientations.

A rough estimate of the total number of vortices is obtained by assuming that the area occupied by a vortex is that of its core, $\pi \xi^{2}$, and for a given area one can fit as many vortex cores until the superconducting state collapses to a normal state. Thus under a parallel field the maximum number of vortices is expected to be $n_{\max }^{\|}=(R / \xi)^{2} \approx 16$, an overestimate of our results (12 vortices). For the perpendicular field this ratio refers to the effective cylinder area, $n_{\max }^{\perp}$ $=2 R D / \pi \xi^{2}: n_{\max }^{\perp s} \approx 10$ and $n_{\max }^{\perp l} \approx 20$. Thus in the case of $H_{\perp}$, this argument predicts that the equal-ratio cylinder can fit twice as many vortices as the short-ratio cylinder, which is 
TABLE I. The matching fields for cylinders with different length, in case of parallel, $H_{L}^{\|}$, and perpendicular, $H_{L}^{\perp}$ fields. Field values are normalized to $H_{c 3}^{\| s}$.

\begin{tabular}{lcccc}
\hline \hline$L \rightarrow L+1$ & $\frac{D=4.0 \xi}{H^{\|}}$ & $\frac{D=4.0 \xi}{H^{\perp}}$ & $\frac{D=8.0 \xi}{H^{\|}}$ & $\frac{D=8.0 \xi}{H^{\perp}}$ \\
\hline 0 & 0.15 & 0.25 & 0.15 & 0.16 \\
1 & 0.26 & 0.37 & 0.26 & 0.26 \\
2 & 0.34 & 0.49 & 0.34 & 0.32 \\
3 & 0.41 & 0.59 & 0.41 & 0.37 \\
4 & 0.49 & 0.68 & 0.49 & 0.46 \\
5 & 0.59 & 0.77 & 0.59 & 0.50 \\
6 & 0.63 & 0.85 & 0.63 & 0.56 \\
7 & 0.69 & 0.93 & 0.69 & 0.61 \\
8 & 0.75 & 1.01 & 0.75 & 0.68 \\
9 & 0.81 & 1.09 & 0.81 & 0.71 \\
10 & 0.88 & & 0.85 & 0.76 \\
11 & 0.96 & & 0.92 & 0.80 \\
12 & & & 0.99 & 1.15 \\
\hline \hline
\end{tabular}

inconsistent with our results of 10 (short-ratio) and 12 (equal-ratio) vortices. We believe that the lateral density depletion, where the short vortices are located, is responsible for rendering $n_{\max }^{\perp s}$ and $n_{\max }^{\perp l}$ lower than expected from this argument. This supports the view that the lateral density depletion is more severe for the equal-ratio than for the short-ratio cylinder.

The intersecting lines in the free energy gives the parallel, $H_{L}^{\|}$, and the perpendicular, $H_{L}^{\perp}$, matching fields for both heights, as summarized in Table I. Interestingly the matching fields for $H_{L}^{\|}$are lower than for $H_{L}^{\perp}$ for the short-ratio but not for the equal-ratio cylinder. The field range over which a $L$ vorticity state is stable is defined as $\Delta H_{L}=H_{L+1}-H_{L}$. Table I shows that for the short-ratio cylinder the perpendicular case displays wider $\Delta H_{L}$ ranges than the parallel case: $\Delta H_{L}^{\perp}$ $>\Delta H_{L}$. This suggests that the perpendicular vortex patterns are more robust than the parallel ones although the Cooper pair density is more depleted at the edges for the perpendicular case than for the parallel one. The maximum number of vortices for the perpendicular case is smaller than for the parallel case because the depletion of the order parameter in the perpendicular case leaves less room for vortices.

The magnetization vs applied field curves are plotted in Fig. 2. Figures $2(\mathrm{e})$ and $2(\mathrm{~g})$ are for the short-ratio and equalratio cylinders, respectively, in the case of $H_{\|}$, and similarly, Figs. 2(f) and 2(h) describe the $H_{\perp}$ case. The rounded upward part of the curves are obtained by increasing the field whereas the lower sawtooth behavior follows by decreasing the field. Thus the red curve is obtained by increasing the field and the blue curve by decreasing the field. These curves show that increasing the field gives a stronger magnetic response than by decreasing it, a signal that increasing the field drives the system through its ground state and decreasing it through excited states. This behavior has been extensively discussed in previous thin-film studies ${ }^{15}$ and here we show that the same picture holds for the perpendicular case.

The highest barrier separating two consecutive states $L$ and $L+1$ happens for $L=1$, for both the $H_{\perp}$ and $H_{\|}$cases. In
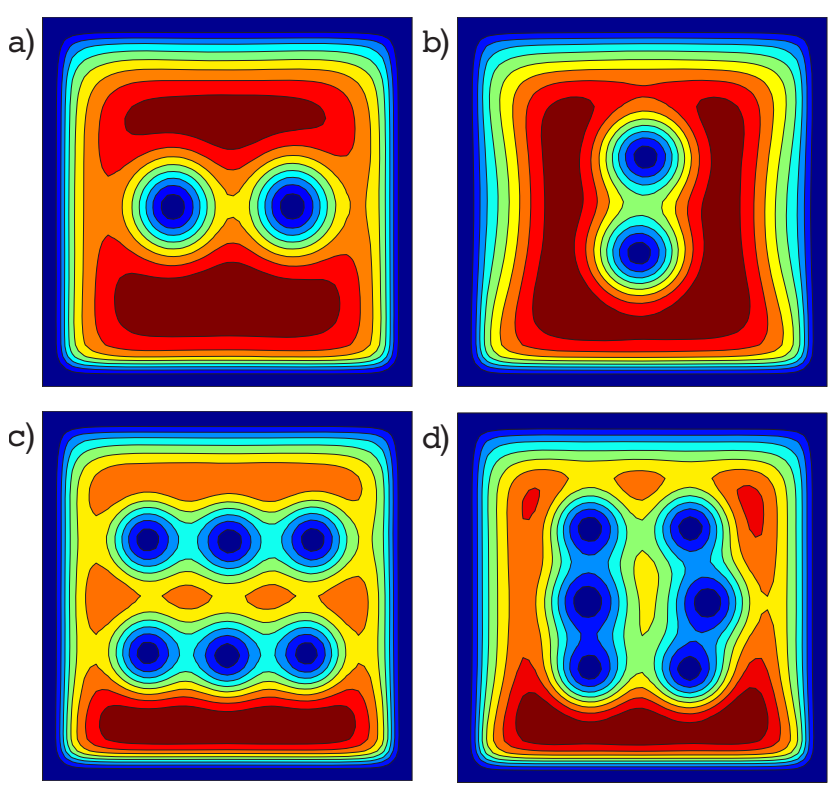

FIG. 3. (Color online) Two-dimensional cross-section density isoplots for the equal-ratio cylinder $(D=8.0 \xi)$ taken in the middle of the disk for applied field $H_{\perp} / H_{\text {ref }}$ equal to $0.226,0.286,0.461$, and 0.512 .

the case of $H_{\|}$this barrier is strong enough to yield a paramagnetic response, as shown in Figs. 2(a) and 2(c) for shortratio and equal-ratio cylinders, respectively. The explanation for this paramagnetic field relies on the energetic barrier which prevents the single vortex to be expelled from the cylinder. This metastable situation lasts to the point that the vortex current surpasses the shielding current present at the cylinder surface. The vortex and the shielding currents are opposite and their sum determines the total magnetization sign, and for a positive sign the situation is paramagnetic. Figures 2(f) and 2(h) show that there is no paramagnetic effect for the perpendicular case as the magnetization is never positive in any of these figures. This is a clear indication that the parallel energetic barrier separating the one and the zero vortex states is higher for the parallel case as compared to the perpendicular case.

In the present extreme type-II limit the magnetization is directly determined from the supercurrent $\vec{J}(\vec{r})$ through $\vec{M}$ $=$ const $\int d v \vec{r} \times \vec{J}(\vec{r})$. The parameter const is determined by imposing that for a small applied field the response is asymptotically linear. Notice that the linear behavior is a signal of the magnetic shielding, or the Meissner effect, which only exists asymptotically in this hard type-II limit. However, this linear regime is important because of the two demagnetization parameters which are expressed in a tensorial condition with two independent equations in the $y-z$ coordinate system whose axis gives

$$
\begin{aligned}
& H_{\|}+4 \pi D_{z z} M_{z}=0, \\
& H_{\perp}+4 \pi D_{y y} M_{y}=0 .
\end{aligned}
$$

From our numerical approach we obtain the ratio between the two nonzero independent demagnetization components, 
TABLE II. Field windows are shown here for the stable states of the short-ratio cylinder. The left column shows expected transitions upon field rotation. Field values are normalized to $H_{c 3}^{\| s}$.

\begin{tabular}{|c|c|c|c|}
\hline $\begin{array}{l}\text { Applied } \\
\text { field range } \\
H\end{array}$ & $\begin{array}{l}\text { Stable no. of } \\
\text { para. vortices } \\
n_{\|}\end{array}$ & $\begin{array}{c}\text { Stable no. of } \\
\text { perp. vortices } \\
n_{\perp}\end{array}$ & $\begin{array}{c}\text { Expected vortex } \\
\text { transition } \\
n_{\|} \rightarrow n_{\perp}\end{array}$ \\
\hline $0.00-0.15$ & 0 & 0 & \\
\hline $0.15-0.25$ & 1 & 0 & $1 \rightarrow 0$ \\
\hline $0.25-0.26$ & 1 & 1 & \\
\hline $0.26-0.34$ & 2 & 1 & $2 \rightarrow 1$ \\
\hline $0.34-0.37$ & 3 & 1 & $3 \rightarrow 1$ \\
\hline $0.37-0.41$ & 3 & 2 & $3 \rightarrow 2$ \\
\hline $0.41-0.49$ & 4 & 2 & $4 \rightarrow 2$ \\
\hline $0.49-0.56$ & 5 & 3 & $5 \rightarrow 3$ \\
\hline $0.56-0.59$ & 6 & 3 & $6 \rightarrow 3$ \\
\hline $0.59-0.63$ & 6 & 4 & $6 \rightarrow 4$ \\
\hline $0.63-0.68$ & 7 & 4 & $7 \rightarrow 4$ \\
\hline $0.68-0.69$ & 7 & 5 & $7 \rightarrow 5$ \\
\hline $0.69-0.75$ & 8 & 5 & $8 \rightarrow 5$ \\
\hline $0.75-0.77$ & 9 & 5 & $9 \rightarrow 5$ \\
\hline $0.77-0.81$ & 9 & 6 & $9 \rightarrow 6$ \\
\hline $0.81-0.85$ & 10 & 6 & $10 \rightarrow 6$ \\
\hline $0.85-0.88$ & 10 & 7 & $10 \rightarrow 7$ \\
\hline $0.88-0.93$ & 11 & 7 & $11 \rightarrow 7$ \\
\hline $0.93-1.00$ & 11 & 7 & $11 \rightarrow 7$ \\
\hline $1.00-1.01$ & & 8 & \\
\hline $1.01-1.12$ & & 9 & \\
\hline
\end{tabular}

$D_{y y} / D_{z z}$, equal to 1.7 and 1.0 for the short-ratio and equalratio cylinders, respectively. Known demagnetizing factor expressions for cylinders ${ }^{25}$ and also for ellipsoids ${ }^{26}$ determine a ratio smaller than 1 for the equal-ratio cylinder. These results apply for a London cylinder $(R, D \gg \lambda)$, whereas here we treat a mesoscopic cylinder $(R, D \ll \lambda)$. In the former case the superconductor's response to the applied field is produced by surface currents, whereas here the current is totally volumetric. The present findings imply that the demagnetization factor should also depend on the ratio between Ginzburg-Landau parameters, $\lambda$ and $\xi$, to the geometrical factors, $R$ and $D$, though this dependence can be safely ignored for macroscopic cylinders. ${ }^{27}$ Hereafter we normalize the magnetization to display this linear Meissner phase, and so, are expressed as $-4 \pi D_{z z} M_{z}$ vs $H_{\|}$and $-4 \pi D_{y y} M_{y}$ vs $H_{\perp}$.

We find here that vortex patterns undergo structural phase transitions for $H_{\perp}$ but not for $H_{\|}$. The states with total vorticity $L=2$ and $L=6$ exhibit this structural transition, related to an abrupt re-positioning of the vortices in the cylinder. The left and right insets of Fig. 2(h) show enlargements of the magnetization curve for the branches $L=2$ and 6, respectively. Two-dimensional contour plots of the vortex patterns associated to this transition are shown in Fig. 3. Figures 3(a) and 3(c) [Figs. 3(b) and 3(d)] show patterns below (above) the structural transition. We explain this transition in terms of the depletion of the Cooper pair density at the edge of the cylinder in the case of $H_{\perp}$. The nucleation of vortices is energetically favorable near the lateral surface because their vortices are shorter than elsewhere. However, the lateral surface also brings to the vortices an opposite effect that pushes them inward. Increasing the field causes the vortices to move from this lateral surface because there the strong Cooper pair density depletion renders them less stable than inside. The structural transition for the simplest case, $L=2$, means that below the transition the two vortices are near the lateral surface, whereas above, they are aligned along the bottom-top direction, i.e., a rotation of $90^{\circ}$ of the vortex configuration.

\section{TILTED FIELD}

A continuous rotation of the applied field unveils interesting features of the vortex patterns, a fact that we show here for the mesoscopic cylinder. These features stem from the different stability regimes of each vorticity $\Delta H_{L}$ taken at the perpendicular and the parallel field directions. Such differences are more pronounced for the short-ratio cylinder and for this reason we concentrate on this case, and show them in Table II, as deduced from Table I. For a fixed value of the

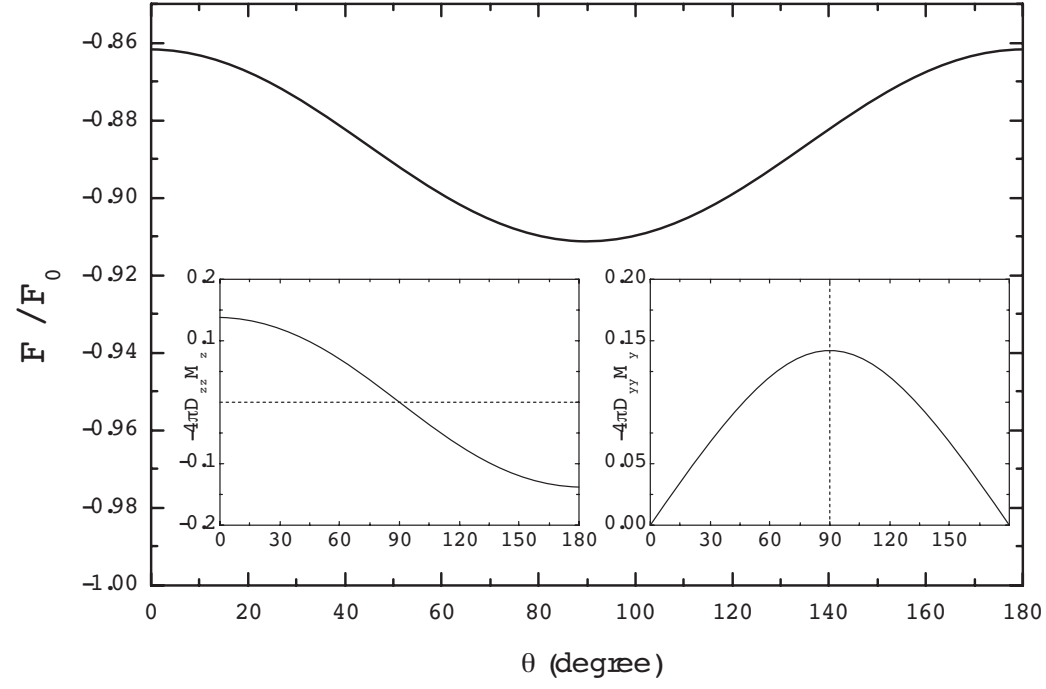

FIG. 4. Free energy for the short-ratio cylinder vs the angle $\theta$ between the magnetic field and the cylinder axis. The applied field is equal to $H / H_{r e f}=0.103$. The insets show the corresponding $z$ and $y$ magnetization. 


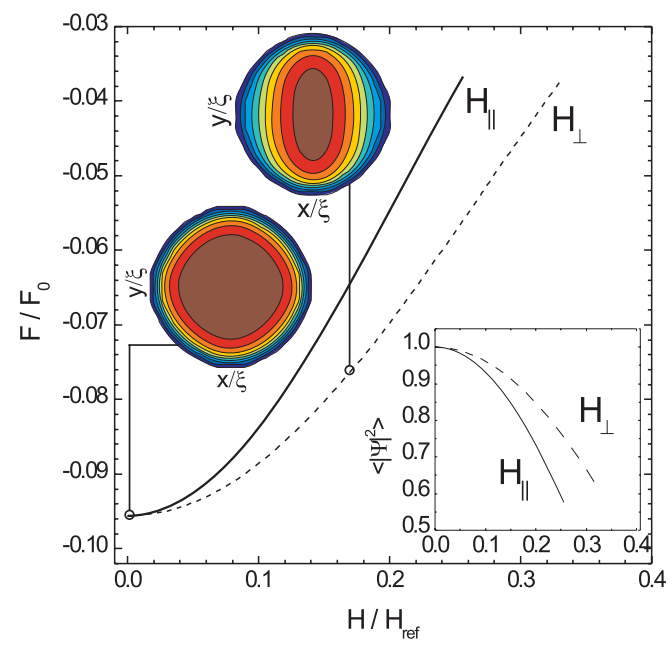

FIG. 5. (Color online) The free energy for the short cylinder vs the applied field for the Meissner phase. The inset shows the average density $\left\langle|\psi|^{2}\right\rangle$ vs the applied field. Dashed and solid lines mean parallel $\left(\theta=90^{\circ}\right)$ and perpendicular $\left(\theta=0^{\circ}\right)$ orientations, respectively.

applied field the number of vortices along the parallel and the perpendicular directions do not necessarily coincide, which implies that upon field rotation vortices freely enter or leave the sample resulting in first-order transition. Table II summarizes these transitions achieved by tilting of the applied field for the short-ratio cylinder.

Here we will discuss the main features we discovered in our numerical study. In order to limit the complexity we will concentrate on the cases which involve at most three vortices. Thus our analysis is restricted to the transitions in the short-ratio cylinder involving zero-to-zero, one-to-zero, oneto-one, two-to-one, three-to-one, and three-to-two regimes. A vortex line connects two surfaces and is in one of the following configurations: bottom-top (bt), bottom-side (bs), sidetop (st), or side-side (ss).

\section{A. Zero-to-zero}

Rotation of the applied field in the regime $0.0<H / H_{\text {ref }}$ $<0.15$ keeps the cylinder in the Meissner phase, according to the first field window of Table II. Considering that the Meissner phase is present in the initial and also in the final states, only geometrical effects are important here. This apparently trivial regime is worth study although it has no vortex nucleation. It turns out that the Meissner phases for parallel and perpendicular directions are different. Figure 4 shows zero-to-zero free energy and magnetization vs $\theta$ curves, taken at field $H / H_{r e f}=0.103$. The free energy associated with the Meissner state for the two field orientations $H_{\|}$ and $H_{\perp}$ are shown in Fig. 5. The lower inset of Fig. 5 shows the average value of the density, $\left\langle|\psi|^{2}\right\rangle$, as a function of the field. Notice that $\left.\left\langle|\psi|^{2}\right\rangle_{\perp}\right\rangle\left\langle|\psi|^{2}\right\rangle_{\|}$throughout the Meissner phase.

We find here that the angular dependence of the free energy of the Meissner phase can be fitted by the function

$$
F \theta, h=F_{0}+h^{p}\left[c+d \sin ^{2} \theta\right],
$$

where the constants $c$ and $d$ are adjustable parameters. This fitting works for the Meissner phase curves of Figs. 4 and 6. Figure 6 also shows zero vortex free energy and magnetization vs $\theta$ curves (black line), taken at field $H / H_{r e f}=0.201$. For the following discussion the field $h$ is a short notation for $H / H_{\text {ref }}$. Let us start with a trial function given by $F(\theta, h)$ $=F_{0}+a(h)+b(h) \sin ^{2}(\theta)$, where $F_{0}$ is an additive constant and there are two functions, $a(h)$ and $b(h)$, that only depend on the magnetic field. Limiting cases help us to deduce these functions. For vanishing field the energy becomes $\theta$ independent and equal to its normalized value, which implies $b(h$ $=0) \rightarrow 0, a(h=0) \rightarrow 0$, and $F_{0}=-1$. For a nonzero parallel field, we have that $F\left(0^{\circ}, h\right)=F_{0}+a(h)$, and this last term is determined from Fig. 2. Similarly, for $\theta=90^{\circ}$, we have that $F\left(90^{\circ}, h\right)=F\left(0^{\circ}, h\right)+b(h)$. Again, this expression is also known from Fig. 2. Since $F\left(0^{\circ}, h\right)$ and $F\left(90^{\circ}, h\right)$ have the same shape and can be fairly well described by an exponential expression proportional to $h^{p}$, we obtain Eq. (4) in terms of field-independent constants $c$ and $d$ that follow from the relation $a(h)=c \cdot h^{p}$ and $b(h)=d \cdot h^{p}$. Since $F\left(90^{\circ}, h\right)$ $<F\left(0^{\circ}, h\right)$ we have the relation $c>0$ and $d<0$. In conclusion we find for the short cylinder that $c=3.347$ and $d=$ -1.202 , which are geometric parameters because they only

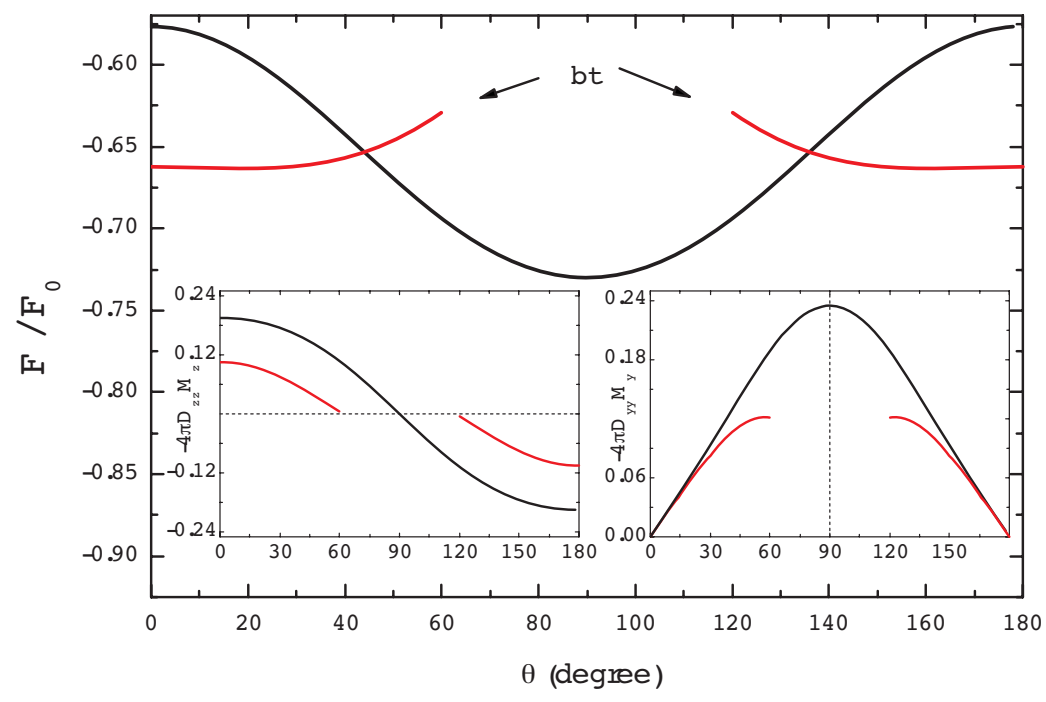

FIG. 6. (Color online) Free energy for the short-ratio cylinder vs the angle $\theta$ between the magnetic field and the cylinder axis. The insets show the corresponding $z$ and $y$ magnetization. The applied field is equal to $H / H_{r e f}=0.201$ and yields a one-vortex $b t$ (red) and a zero-vortex (black) state. 

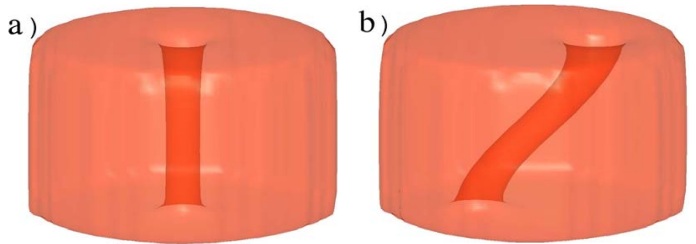

FIG. 7. (Color online) Isosurface of the density taken at $|\psi(\vec{r})|$ $=0.2$ for an applied field equal to $H / H_{\text {ref }}=0.201$ and (a) $\theta=0^{\circ}$ and (b) $59^{\circ}$.

depend on the field orientation with respect to the axis. The choice of just one single exponent $p$ is sufficiently accurate to describe both functions $a(h)$ and $b(h)$. A simultaneous fit of the parallel and perpendicular curves gives $p=1.68$.

The Cooper pair density in the Meissner states for $H_{\perp}$ and $H_{\|}$is different. For $H_{\perp},|\psi|^{2}$ is depleted in the lateral surface, near the contact straight lines, as compared to the bottom and top surfaces. This depletion somehow increases the density in the center-the plane of rotation $(y-z)$. This explains the lower free energy for $H_{\perp}$ as compared to $H_{\|}$, as shown in Fig. 5.

\section{B. One-to-zero}

A continuous tilt of the angle starting from $\theta=0^{\circ}$, in the case of one vortex, is interesting because the vortex must be expelled at some critical angle where the Meissner phase sets in. The one-vortex state lives in different field windows, for the parallel case, $0.15<H_{\|} / H_{r e f}<0.26$, and for the perpendicular case, $0.25<H_{\perp} / H_{r e f}<0.37$, as shown in Table I. No perpendicular vortex is possible in the ground state for $0.15<H_{\perp} / H_{r e f}<0.25$, although it may exist as a metastable state. This is the second field window shown in Table I.

Figure 6 shows for $H / H_{r e f}=0.201$ the free energy vs the direction of the applied field, $\theta$. For $0^{\circ}$ the one-vortex and the zero-vortex states correspond to the ground and first excited states, respectively. Tilting the applied field causes opposite effects to these states: the single-vortex state grows in energy and the zero-vortex state decreases in energy and for $\theta \cong 44^{\circ}$ the two lines cross each other. For this angle the parallel field, $H^{\|} / H_{r e f}=0.201 \cos \left(44^{\circ}\right)=0.14$, falls into the first range described in Table II, which means that the zerovortex state, and not the one-vortex state, is stable. For angles larger than $60^{\circ}$ our numerical procedure is no longer able to find a metastable (one-vortex) state and only the ground (zero-vortex) state is obtained. Figure 7 shows threedimensional isodensity plots for fixed applied field, $\mathrm{H} / \mathrm{H}_{\text {ref }}$ $=0.201$, taken at (a) $\theta=0^{\circ}$ and (b) $59^{\circ}$. This is the maximum possible angle before the expulsion of the vortex. These two points correspond to the first and the last points of the $b t$ branch of Fig. 6. Notice that the vortex is oriented along the field in both cases but is deformed in the tilted case. The vortex must reach the bottom and top surfaces perpendicularly because there should be no current component normal to the surface.

\section{One-to-one}

In this small field window, $0.25<H / H_{r e f}<0.26$, the single vortex remains inside the superconductor upon field rotation. This apparently straightforward regime reveals two transitions by increasing the angle, as the vortex line partially hops from a bottom-top line to a side-top line. The second transition is a hop to a side-side line. Figure 8 shows the free-energy and magnetization curves, which display reflection symmetry around $90^{\circ}$, as expected, and three distinct branches, associated to bottom-top, side-top, or bottom-side, and side-side (see Fig. 9). We have performed a field sweep ranging from $0^{\circ}$ to $180^{\circ}$, with steps of $0.5^{\circ}$, for a fixed applied field $H / H_{r e f}=0.255$. The free energy decreases for increasing angle but this trend is not generally followed for $H / H_{r e f}=0.255$, as shown in Fig. 8. The first free-energy branch $(b t)$ has a minimum for $\theta=43^{\circ}$, and not for $\theta=0^{\circ}$, which corresponds to a local maximum, a quite surprising and peculiar result. A tilted vortex has lower energy than the straight vortex. The second branch $(s t)$ ranges from $\theta=58^{\circ}$ to $\theta=73^{\circ}$ and corresponds to a side-top or bottom-side vortex line configuration. This second branch is stable over an an-

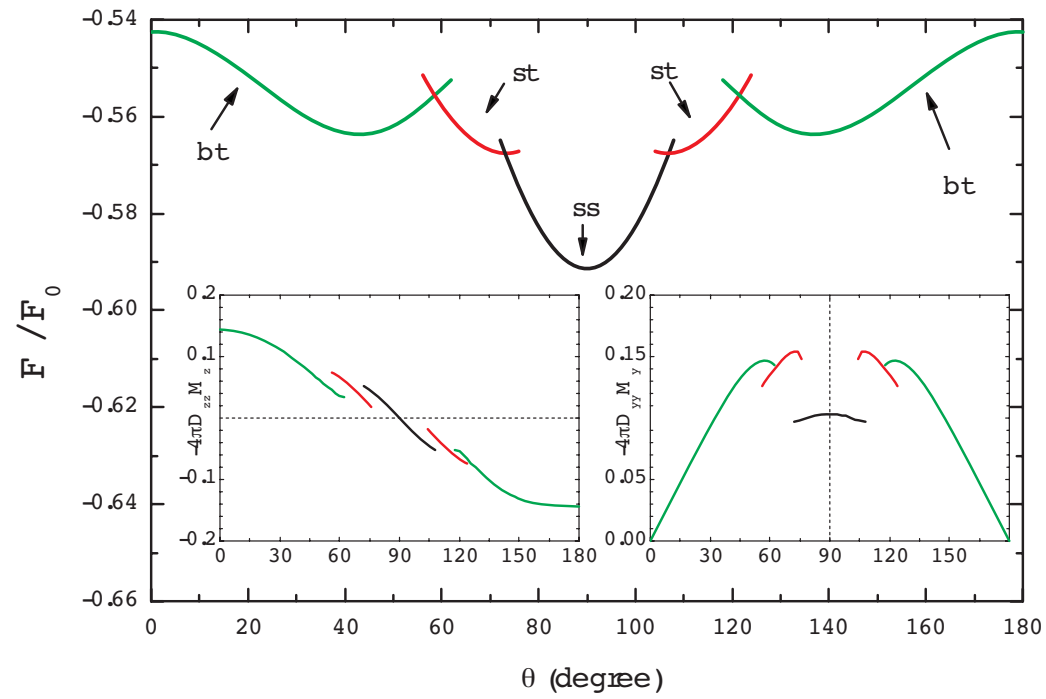

FIG. 8. (Color online) Free energy for the short-ratio cylinder vs the angle $\theta$ between the magnetic field and the cylinder axis. The insets show the corresponding $z$ and $y$ magnetization. The applied field is equal to $H / H_{r e f}=0.255$ and yields a bottom-top (green), a side-top (red), or bottom-side, and side-side (black) state upon rotation. 

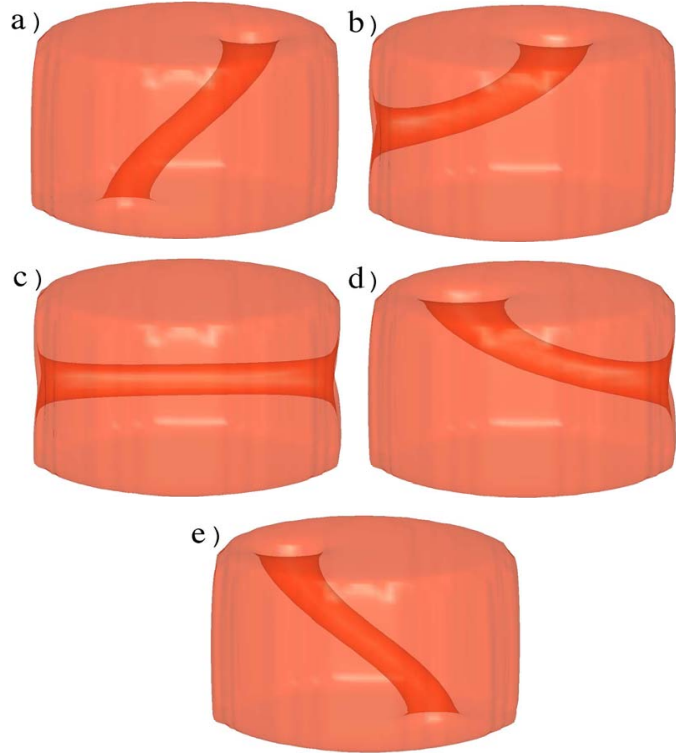

FIG. 9. (Color online) Density isosurfaces taken at $|\psi(\vec{r})|^{2}=0.2$ are shown here. They correspond to an applied field equal to $H / H_{\text {ref }}=0.255$ and angle equal to (a) $56^{\circ}$, (b) $70^{\circ}$, (c) $88^{\circ}$, (d) $104^{\circ}$, and (e) $120^{\circ}$, respectively.

gular range of $15^{\circ}$. Next is the third branch $(s s)$, the central region where the side-side vortex state is found. It exhibits the lowest energy among all the regions. The angular range for this region is of $34^{\circ}$. Though the central region shows the lowest energy, the bottom-top region has the largest angular range of stability. The explanation for the range of stability of the three branches probably relies on possible ways to accommodate the vortex upon rotation. In bottom-top, the vortex has more "space" to change its position than in sideside.

We interpret the free-energy jumps of Fig. 8 as first-order structural transitions very much in the sense of the previously studied transition of Fig. 3. However, the transitions of Fig. 8 are associated to jumps of the quantum flux. The vortex cannot move continuously from one surface to the other without producing a discontinuity of the flux in a given surface (see Fig. 9).

\section{Two-to-one}

The case of a rotating applied field within the fourth field window of Table II has two vortices for $\theta=0^{\circ}$ and only one for $\theta=90^{\circ}$. In this regime there are many possible initial configurations, since any orientation of the plane containing the two vortices with respect to the $y-z$ plane is possible. Field rotation along the $y-z$ plane lifts these degeneracies which means that the free energy vs $\theta$ is no longer universal. It is also possible to have a $(L=2)$ giant vortex within the upper part of this field window. Generally speaking giant vortex and multivortex states are found in different sectors of a single $L$ curve. The giant vortex splits into two vortices for a very small tilt and proceeds from there on as a two-vortex state.

Figure 10 shows that the two-vortex state (dashed gray blue line) lasts until the maximum angle of $38^{\circ}$, and from there on, one vortex exits and only one vortex is found in the bottom-top, side-top, and side-side configurations. Figure 11 shows three-dimensional isoplots and two-dimensional contour plots (top views) for $H / H_{r e f}=0.28$ and the extreme angles of $\theta=0^{\circ}$ (a) and of $38^{\circ}$ (b).

\section{E. Three-to-one}

The fifth field window of Table II gives rise to more elaborate free-energy and magnetization diagrams than the previous ones. Figure 12 shows the free-energy and magnetization curves for $H / H_{r e f}=0.35$, which upon rotation decays according to the sequence $3 b t \rightarrow 2 b t \rightarrow 1 b t+1 s t \rightarrow 1 s t+1 b s$ $\rightarrow 1$ ss. The insets show their respective $z$ and $y$ magnetization. Notice that the three-to-one transition state passes through two-vortex states. Some intermediate states ( $2 b t$ and $b t+s t)$ show the remarkable feature previously found, of the local free-energy minimum taking place at some tilt angle away from the minimum $\theta$.

There are many three-vortex states for $\theta=0^{\circ}$ and rotation

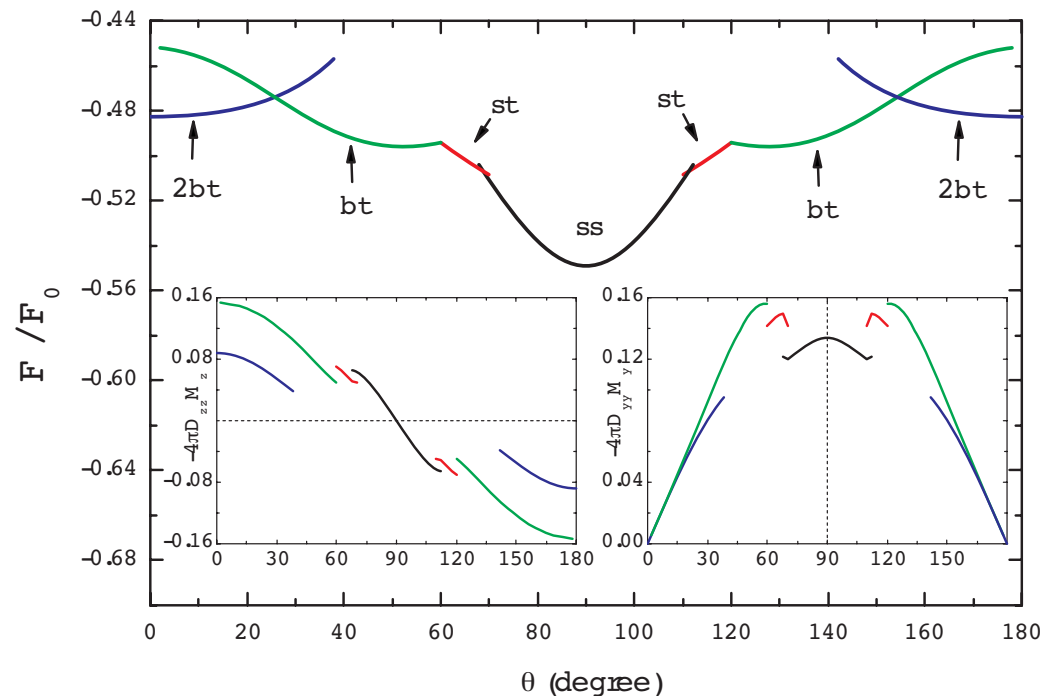

FIG. 10. (Color online) Free energy for the short-ratio cylinder vs the angle $\theta$ between the magnetic field and the cylinder axis. The insets show the corresponding $z$ and $y$ magnetization. The applied field is equal to $H / H_{r e f}=0.28$ and leads upon rotation to $2 b t$ (blue), bt (green), st (red), and ss (black) states. 

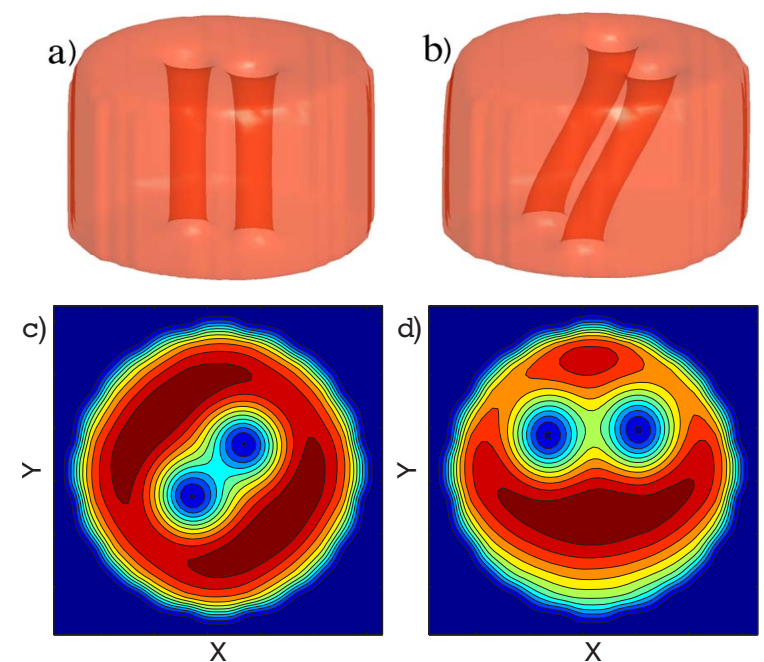

FIG. 11. (Color online) (a), (b) Three-dimensional isoplot for the short-ratio cylinder taken at $|\psi(\vec{r})|^{2}=0.2$ for $H / H_{\text {ref }}=0.28$ and (a) $\theta=0$ and (b) $38^{\circ}$. The corresponding contour plots taken at the top surface are shown in (c), (d). The blue to red hue means minimum to maximum density values.

along the $y-z$ plane is slightly different for each one of them and this produces small energy differences which are not discussed here.

\section{F. Three-to-two}

Higher field windows lead to a wealth of vortex configurations and cascades between them. Figure 13 shows the angular dependency for the free energy for a field that falls in the sixth window described in Table II. The vortex configuration cascades from $\theta=0^{\circ}$ to $90^{\circ}$ into a sequence of states given by $3 b t \rightarrow 2 b t+b s \rightarrow s t+b t+b s \rightarrow s t+2 b s \rightarrow s t+s s+b s$ $\rightarrow 2 s s$. Distinctly from all previous cases, here a $s s$ appears together with a $b s$ or $s t$ state, proving their coexistence, as shown in Fig. 13.

Last we remark that the transitions displayed in Figs. 4, 6, $8,10,12$, and 13 reflect the diameter-to-thickness aspect ratio of the short cylinder and are expected to change accordingly. For very tall cylinders $(D \gg R)$ a side-side state is much less costly than a bottom-top state, just because such a vortex is much shorter. Consequently one expects a larger discontinuity separating these two states and smaller angular windows in this limit.

\section{VORTEX LENGTH ANALYSIS}

The small volume-to-surface ratio of mesoscopic superconductors makes bulk and surface effects important in fixing the length of the vortex line, as shown here. We have directly obtained the vortex length from our numerical results and compared it with three simple models that give the following expressions for the vortex line:

$$
l_{1}(\theta)=D / \cos \theta
$$

$$
l_{2}(\theta)=D / \cos \theta+2 \xi(1-1 / \cos \theta)
$$

$$
l_{3}(\theta)=D\left[1+(1-2 \xi / D)^{2} \tan ^{2} \theta\right]^{1 / 2} .
$$

The first model consists of a straight line passing through the center of the cylinder and parallel to the field. Its length $l_{1}(\theta)$

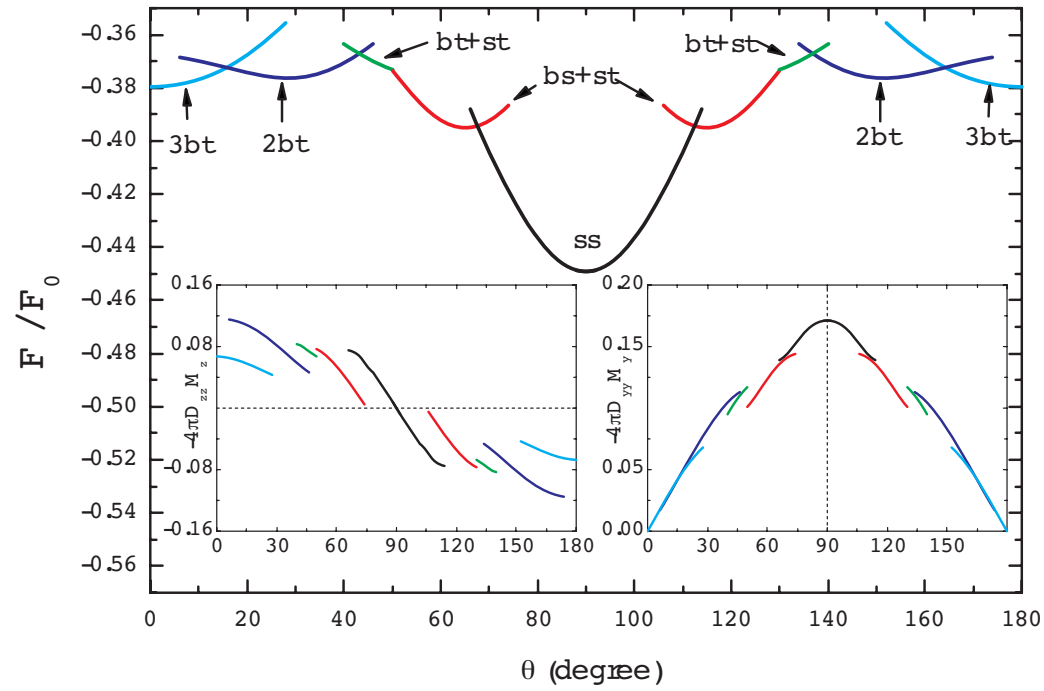

a

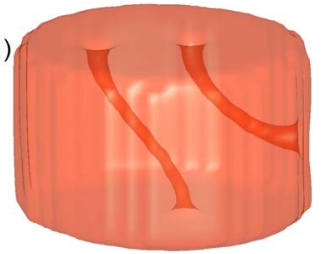

b)

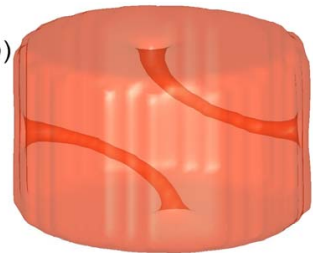

FIG. 12. (Color online) Free energy for the short-ratio cylinder vs the angle $\theta$ between the magnetic field and the cylinder axis. The insets show the corresponding $z$ and $y$ magnetization. The applied field is equal to $H / H_{r e f}=0.35$ and leads upon rotation to $3 b t$ (cyan), $2 b t$ (blue), $b t$ $+s t$ (green), $b s+s t$ (red), and $s s$ (black) states. We show three-dimensional isosurface plots in (a) for the $b t+s t$ state $\left(\theta=44^{\circ}\right)$ and in (b) for the $b s+s t$ state $\left(\theta=64^{\circ}\right)$, taken at $|\psi(\vec{r})|^{2}=0.05$. 


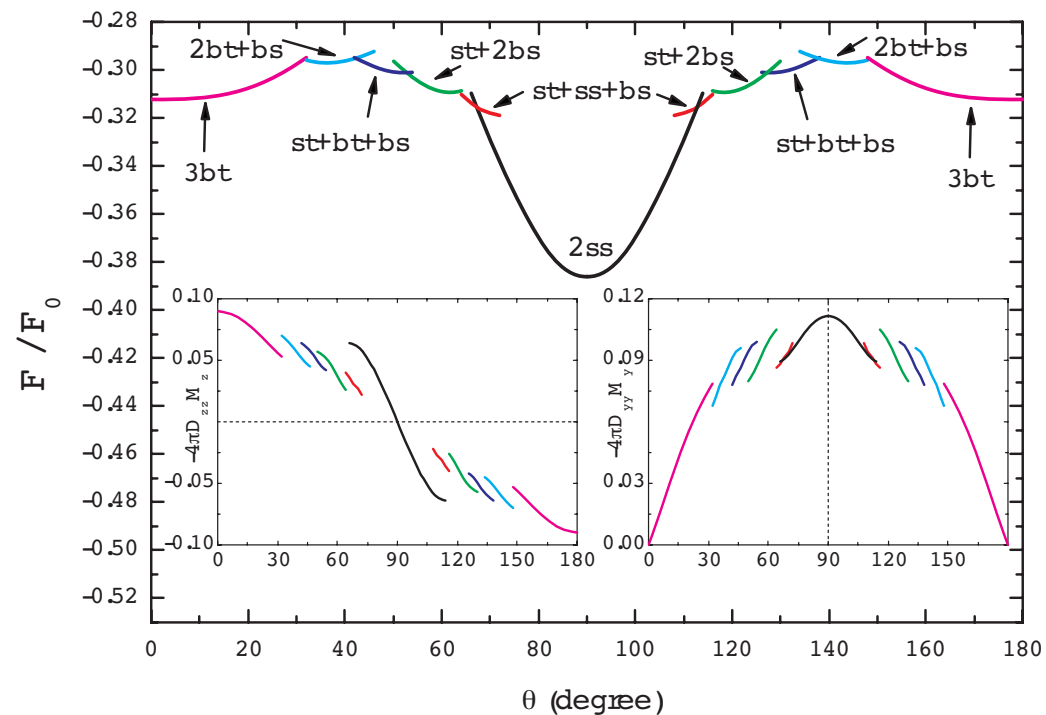

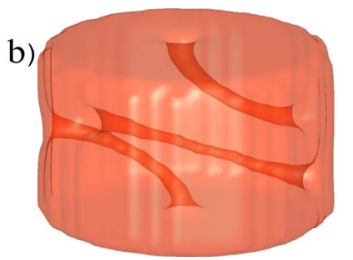

FIG. 13. (Color online) Free energy for the short-ratio cylinder vs the angle $\theta$ between the magnetic field and the cylinder axis. The insets show the corresponding $z$ and $y$ magnetization. The applied field is equal to $H / H_{r e f}=0.40$ and leads upon rotation to $3 b t, 2 b t+b s$ (red), $s t+b t$ $+b s$ (cyan), $s t+2 b s$ (blue), $s t+s s+b s$ (green), $s t$ $+s s+b s$ (red), and 2ss (black). We show threedimensional isosurfaces for (a) the $s t+b t+b s(\theta$ $\left.=42^{\circ}\right)$ and (b) the $s t+b t+b s\left(\theta=64^{\circ}\right)$ states, taken at $|\psi(\vec{r})|^{2}=0.05$. reaches a maximum for $\theta=a \tan (2 R / D) \cong 63.4^{\circ}$, in the case of the short-ratio cylinder. The second model differs from the first near the surface because the straight lines break at a distance $\xi$ to emerge perpendicular to the bottom and top surfaces. This line length $l_{2}(\theta)$ is a sum of three segments, two with size $\xi$ and the central one with length $(D$ $-2 \xi) / \cos (\theta)$. The third model $l_{3}(\theta)$ is just a straight line joining the two extremities of the second model line. It is oriented along $\theta^{\prime}$ instead, defined by $\tan \theta^{\prime}=(1$ $-2 \xi / D) \tan \theta$. This is a useful model when regarded as a vortex line with a vanishing entrance segment, instead of $\xi$, as for the second model. Numerically we determine the length of the vortex line by first locating the position of the core in the bottom and top surfaces. Then we proceed to calculate the length of the vortex in two ways. First, we consider the $(x, y)$ position of the minimum of $|\psi|^{2}$ in every layer along the $z$ direction. Connecting the mesh points, a total of 48 points, we find the length of the vortex. Second, we connect the vortex cores at the two surfaces and join them by the shortest straight line. These procedures are called three-dimensional (3D) length and short length, respectively. We present all the models and numerical curves in Fig. 14: the reference length (solid blue curve), the 3D length (dashed black curve), and the short length (dotted red curve). At low angles, near zero, all curves converge to the same value, the height of the cylinder, $D=4 \xi$. In the range $4^{\circ} \leqslant \theta \leqslant 24^{\circ}$, the 3D length shows a larger growth rate than the other two basically because of the deformation near the flat surfaces. For large angles, $32^{\circ} \leqslant \theta \leqslant 60^{\circ}$, the difference between the short length and the 3D length is reduced. Asymptotically, these last two lengths should converge to the same values at $\theta=90^{\circ}$, but the state bottom-top is not allowed for angles larger than $63.4^{\circ}$. In summary this compari- son shows very clearly that oblique vortices emerge perpendicular to the surface over a distance characterized by $\xi$.

\section{CONCLUSIONS}

We have shown, using a three-dimensional approach to solve the Ginzburg-Landau theory, that vortex patterns for a mesoscopic superconducting cylinder show unique features caused by their finite length. Two cylinders with intermediate ratio to diameter were considered and several of their properties were determined, such as critical and matching fields

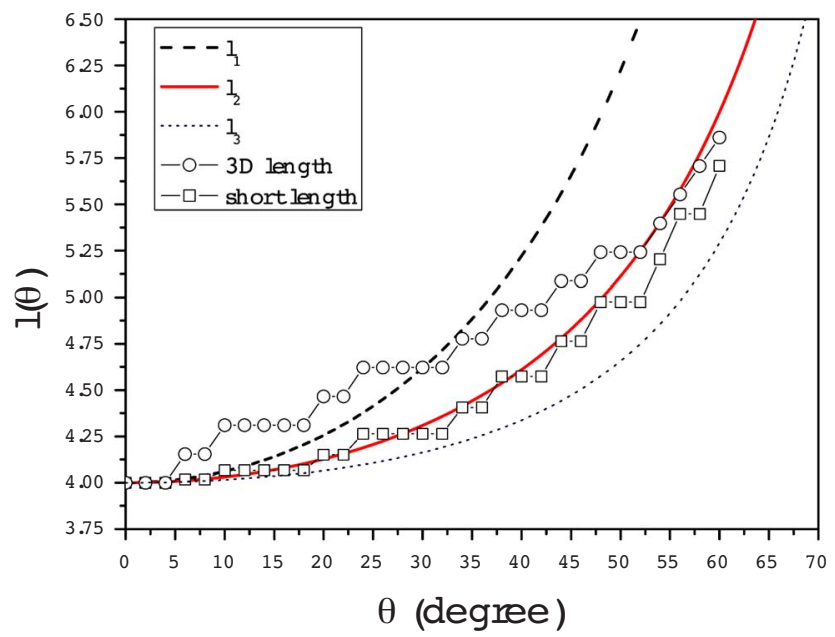

FIG. 14. (Color online) Vortex length as a function of the field orientation. The modulus of the field is kept constant and equal to $H / H_{\text {ref }}=0.255$ while the angle $\theta$ changes from $0^{\circ}$ to $180^{\circ}$. The solid line is the vortex length and the dashed line is the straight line. 
for parallel and perpendicular fields. Structural transitions were found to exist in the case of a perpendicular field. Below the transition the vortices stay near to the lateral surface because their length is shorter there, but they are forced to migrate to the center as their arrangement becomes unstable due to the density depletion near the lateral surface. The different field windows of existence of a particular vorticity configuration makes the rotation of the applied field produce remarkable features. Because of the small volume-to-surface ratio the tilted field brings conflicting energetic demands to the vortex lines, which must remain oriented along the applied field and emerge perpendicular to the surface. We have analyzed several transitions found upon tilting in the case of zero, one, two, and three vortices. Interestingly we have shown here that for some fixed value of the applied field it is possible to find a tilted line with lower energy than a line oriented along the major axis.

We found that with increasing tilt of the magnetic field away from the cylinder axis (i) first a giant vortex (if it exists) splits into multiple lines, then (ii) the vortex lines start to tilt such that in the middle of the cylinder they are aligned with the applied field. With further tilt of the magnetic field (iii) a phase transition occurs where one of the vortices leaves the sample or, depending on the field strength, the edge of one of the vortices jumps to the side of the cylinder resulting in a side-top vortex state. Later this vortex will be aligned to $90^{\circ}$. Subsequently, if other vortices are present, (iv) the edge of another vortex may jump to the opposite side of the cylinder resulting in a bottom-side vortex. Then there are different possibilities, if not more than two vortices are present, (v) the edge of one of the bent vortices will jump such that one obtains a vortex that is almost parallel to the top and bottom surfaces of the cylinder. Depending on the magnetic-field value it is also possible that before the latter jump happens one of the vortices leaves the sample. When more than two vortices are present there is another possibility which may happen before, namely, (vi) a bottom-top may jump to a vortex that is almost parallel to the top surface of the cylinder. The above scenarios are continued until all vortices are directed parallel to the surface of the cylinder. It is clear that if for $\theta=0^{\circ}$ the vorticity is large a very rich series of possible phase transitions should be experimentally observed by measuring the magnetization of the sample using a superconducting quantum interference device ${ }^{28}$ or a microHall magnetometer ${ }^{29,30}$ where the whole experimental setup is tilted with respect to external magnetic field. The stability of the different tilted vortex configuration can be traced out by measuring the magnetization with increasing and decreasing tilt of the magnetic field.

\section{ACKNOWLEDGMENTS}

A.R.C.R acknowledges the Brazilian agency CNPq for financial support. M.M.D. thanks CNPq, FAPERJ, Instituto do Milênio de Nanotecnologia (Brazil), and BOF/UA (Belgium). F.M.P. acknowledges support from the Flemish Science Foundation (FWO-Vl), the Belgian Science Policy (IUAP), and the ESF-AQDJJ network.
*Electronic address: ton@if.ufrj.br

${ }^{\dagger}$ Electronic address: mmd@if.ufrj.br

Electronic address: francois.peeters@ua.ac.be; URL: http:// www.cmt.ua.ac.be

${ }^{1}$ A. K. Geim, I. V. Grigorieva, S. V. Dubonos, J. G. S. Lok, J. C. Maan, A. E. Filippov, and F. M. Peeters, Nature (London) 390, 259 (1997).

${ }^{2}$ A. K. Geim, S. V. Dubonos, I. V. Grigorieva, K. S. Novoselov, F. M. Peeters, and V. A. Schweigert, Nature (London) 407, 55 (2000).

${ }^{3}$ P. S. Deo, V. A. Schweigert, F. M. Peeters, and A. K. Geim, Phys. Rev. Lett. 79, 4653 (1997).

${ }^{4}$ V. A. Schweigert, F. M. Peeters, and P. S. Deo, Phys. Rev. Lett. 81, 2783 (1998).

${ }^{5}$ B. J. Baelus, F. M. Peeters, and V. A. Schweigert, Phys. Rev. B 63, 144517 (2001).

${ }^{6}$ I. V. Grigorieva, W. Escoffier, J. Richardson, L. Y. Vinnikov, S. Dubonos, and V. Oboznov, Phys. Rev. Lett. 96, 077005 (2006).

${ }^{7}$ A. Kanda, B. J. Baelus, F. M. Peeters, K. Kadowaki, and Y. Ootuka, Phys. Rev. Lett. 93, 257002 (2004).

${ }^{8}$ L. F. Chibotaru, A. Ceulemans, V. Bruyndoncx, and V. V. Moshchalkov, Nature (London) 408, 833 (2000).

${ }^{9}$ R. Geurts, M. V. Milosevic, and F. M. Peeters, Phys. Rev. Lett. 97, 137002 (2006).

${ }^{10}$ B. J. Baelus, S. V. Yampolskii, F. M. Peeters, E. Montevecchi, and J. O. Indekeu, Phys. Rev. B 65, 024510 (2001).
${ }^{11}$ A. K. Elmurodov, D. Y. Vodolazov, and F. M. Peeters, Europhys. Lett. 74, 151 (2006).

${ }^{12}$ J. B. Ketterson and S. N. Song, Superconductivity, 1st ed. (Cambridge University Press, Cambridge, England, 1999).

${ }^{13}$ P. G. de Gennes, Superconductivity in Metals and Alloys, 2nd ed. (Persus Book, 1989).

${ }^{14}$ Q. Du, J. Math. Phys. 46, 095109 (2005).

${ }^{15}$ B. J. Baelus and F. M. Peeters, Phys. Rev. B 65, 104515 (2002).

${ }^{16}$ E. H. Brandt, J. Low Temp. Phys. 42, 557 (1981).

${ }^{17}$ A. R. de C. Romaguera and M. M. Doria, Eur. Phys. J. B 42, 3 (2004).

${ }^{18}$ M. M. Doria and A. R. de C. Romaguera, Europhys. Lett. 67, 446 (2004).

${ }^{19}$ M. M. Doria, A. R. de C. Romaguera, and F. M. Peeters, Phys. Rev. B 75, 064505 (2007).

${ }^{20}$ P. S. Deo, V. A. Schweigert, and F. M. Peeters, Phys. Rev. B 59, 6039 (1999).

${ }^{21}$ M. N. Kunchur and S. J. Poon, Phys. Rev. B 43, 2916 (1991).

${ }^{22}$ J. J. Palacios, Phys. Rev. Lett. 84, 1796 (2000).

${ }^{23}$ V. A. Schweigert and F. M. Peeters, Phys. Rev. Lett. 83, 2409 (1999).

${ }^{24}$ J. J. Palacios, F. M. Peeters, and B. J. Baelus, Phys. Rev. B 64, 134514 (2001).

${ }^{25}$ S. Tandon, M. Beleggia, Y. Zhu, and M. D. Graef, J. Magn. Magn. Mater. 271, 9 (2004). 
${ }^{26}$ J. A. Osborn, Phys. Rev. 67, 351 (1945).

${ }^{27}$ F. M. Araujo-Moreira, C. Navau, and A. Sanchez, Phys. Rev. B 61, 634 (2000).

${ }^{28}$ C. Veauvy, K. Hasselbach, and D. Mailly, Rev. Sci. Instrum. 73, 3825 (2002).
${ }^{29}$ A. K. Geim, S. V. Dubonos, J. G. S. Lok, M. Henini, and J. C. Maan, Nature (London) 396, 144 (1998).

${ }^{30}$ A. K. Geim, S. V. Dubonos, J. G. S. Lok, I. V. Grigorieva, J. C. Maan, L. T. Hansen, and P. E. Lindelof, Appl. Phys. Lett. 71, 2379 (1997). 